\title{
Neuer Vorstand in der Deutschen Gesellschaft für Lymphologie
}

Frau Dr. Anya Miller, Präsidentin der Deutschen Gesellschaft für Lymphologie hat uns das Wahlergebnis und die Besetzung des neuen Vorstands mitgeteilt, die wir gerne an unsere Leserschaft weitergeben:

Präsidentin: Dr. Anya Miller Generalsekretärin: Dr. Barbara Netopil Geschäftsführer: Ralph Martig
Sprecher der physiotherapeutischen Berufsgruppen: Thomas Künzel

Schriftführerin: Dr. Gabriele Faerber

Vorsitzender des Schulungsausschusses

Dr. Christina Schreiber

Beisitzer aus dem Kreis der Lymphdrainage

und Ödemtherapie: Oliver Gültig
Hautschriftleiter der Zeitschrift: Prof. Dr. Erich Brenner

Beisitzer aus dem Kreis der Ästhetischen Lymphologie: Dr. Yvonne Frambach Beisitzer aus dem Kreis der operativen Lymphologie: Dr. Mario Marx

Wissenschaftlicher Beirat: Prof. Hellmuth Zöltzer, Dr. Yvonne Frambach Ehrenpräsident: Prof. Horst Weissleder 